\title{
Grace Wahba and the Wisconsin Spline School
}

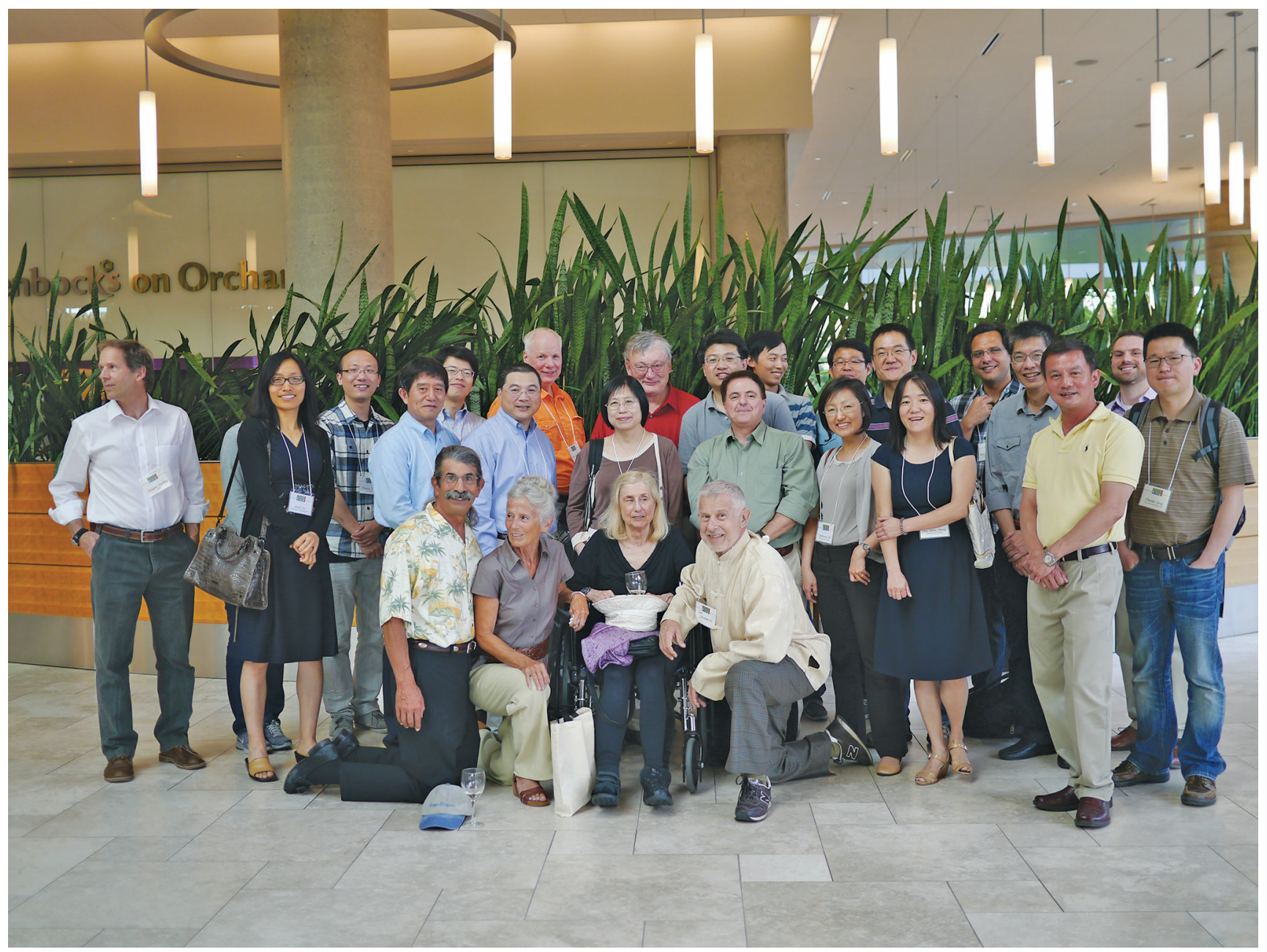

\section{Chong Gu and Yuedong Wang}

Chong Gu is a professor of statistics at Purdue University. His email address is chong@purdue.edu.

Yuedong Wang is a professor of statistics at the University of California, Santa Barbara. His email address is yuedong@pstat. ucsb. edu.

The opening photo is from a conference in honor of Wahba, 2014. Front from left: Scott Gamlen*, Linda Gamlen*, Grace Wahba, Sydney Goldsmith*. Middle from left: Anna Liu (UMass Amherst), Zehua Chen (NUS), Yuedong Wang (UCSB), Jyh-Jen Shiau (National Chiao Tung U, Taiwan), Ronaldo Dias (State U of Campinas, Brazil), Yoonkyung Lee (Ohio State), Helen Zhang (U Arizona), Wing Hung Wong (Stanford), Wensheng Guo (UPenn), Chenlei Leng (Warwick). Back from left: Doug Nychka (Colorado School of Mine), obscured, Zhigeng Geng (Apple), Yufeng Liu (UNC), Jim Wendelberger (Los Alamos Lab, U New Mexico), David Callan*, Dong Xiang (Amazon), Bin Dai
Grace Wahba (née Goldsmith, born August 3, 1934) joined the Department of Statistics at the University of Wisconsin-Madison in 1967, and remained on its faculty until her retirement in 2019. During her luminous career

(Tower Research Capital), Shilin Ding (Facebook), Ming Yuan (Columbia), Héctor Corrada Bravo (Genetech), Robert Krafty (Emory). Names with* are Grace's family members. All of the rest are Grace's academic descendants followed by current affiliations.

Communicated by Notices Associate Editor Richard Levine.

For permission to reprint this article, please contact:

reprint-permission@ams.org.

DOI: https://doi.org/10.1090/noti2438 
at Madison of more than half a century, Grace graduated 39 doctoral students and has over 400 academic descendants to date. Over the years, Grace has worked on countless interesting problems, covering a broad spectrum from theory to applications. Above all, Grace is best known as the mother of the Wisconsin spline school, the primary driving force behind a rich family of data smoothing methods developed by herself, collaborators, and generations of students. Some of Grace's life stories can be found in a recent entertaining piece by Nychka, Ma, and Bates [10]. Here, we try to complement that piece with some technical discussions concerning the Wisconsin spline school.

As Grace's former students, our first set of reading assignments consisted of the defining document of reproducing kernel Hilbert spaces by Aronszajn [1] and three early papers by Kimeldorf and Wahba [4-6]. As we shall demonstrate below, some of those early results had far-reaching impacts on developments decades later. According to [10], many of the ideas of Kimeldorf and Wahba were inspired by discussions during tea parties at the Mathematics Research Center at UW Madison in the late 1960s/early 1970s, with participants including Issac Schoenberg, Carl de Boor, and Larry Schumaker.

In the sections to follow, we shall outline the smoothing spline approach to data smoothing, addressing numerous aspects and noting similarities and differences compared to related techniques. We highlight Grace's many original contributions, but otherwise focus on the flow of presentation; for more accurate attributions of credit, one may consult the forward of [16] and the bibliographic notes in [3].

\section{Smoothing Splines}

Given pairs $\left(x_{i}, y_{i}\right), i=1, \ldots, n, x_{i} \in[a, b]$, one may obtain a smoothing spline via the minimization of

$$
\frac{1}{n} \sum_{i=1}^{n}\left(y_{i}-\eta\left(x_{i}\right)\right)^{2}+\lambda \int_{a}^{b}\left(\eta^{\prime \prime}(x)\right)^{2} d x .
$$

The minimizer of (1) is a piecewise cubic polynomial, three times differentiable, with the third derivative jumping at the distinctive $x_{i}$ points.

In the mathematics or numerical analysis literature, a spline typically refers to a piecewise polynomial, one is concerned with function interpolation or approximation, and the $\left(x_{i}, y_{i}\right)$ pairs are exact samples satisfying $y_{i}=\eta\left(x_{i}\right)$.

With stochastic data, one does not have exact samples of the function and needs statistical models. A regression model behind (1) has $y_{i}=\eta\left(x_{i}\right)+\epsilon_{i}, \epsilon_{i} \sim N\left(0, \sigma^{2}\right)$ independent, and the minimizer of (1) provides an estimate of the regression function $\eta(x)$.

The first term in (1) pushes for a close fit of $\eta$ to the data and the second term penalizes the roughness of $\eta$, with the smoothing parameter $\lambda$ controlling the tradeoff between

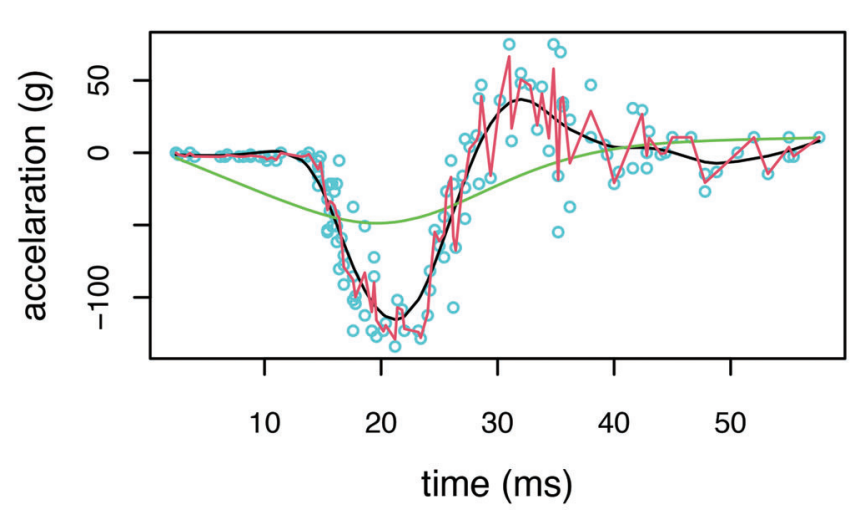

Figure 1. Cubic smoothing splines.

the two conflicting goals. As $\lambda \rightarrow \infty$, one approaches a simple linear regression line $\eta(x)=\beta_{0}+\beta_{1} x ; \lambda=0_{+}$yields the minimum curvature interpolant.

Figure 1 illustrates the cubic smoothing spline of (1) applied to some data from a simulated motorcycle accident, found in R package MASS as data frame mcycle, where $x$ is time and $y$ is head acceleration. The data are in circles and the three lines correspond to $\log _{10} n \tilde{\lambda}=-8.5,-4.08,-1.5$, from rough to smooth (red, black, and green, respectively), with $\log _{10} n \tilde{\lambda}=-4.08$ (black color) selected by cross validation via (15) using ssanova 0 in $\mathrm{R}$ package gs $; \lambda / \tilde{\lambda}=60^{3}$ accounts for the mapping of $[0,60]$ onto $[0,1]$ in the software implementation.

Regression analysis, a primary tool of supervised learning, is widely used in applications. Traditional parametric regression was developed when data were scarce, restricting $\eta(x)$ to low-dimensional function spaces with a few free parameters; this maintains sufficient samples per parameter to effectively control "variance" in estimation, but may incur serious model bias. As the sample size $n$ increases, "variance" is less of a concern and one looks to reduce model bias, yielding numerous non-parametric regression techniques that permit "flexible" forms of $\eta(x)$. The smoothing spline of (1) presents a tidy approach to non-parametric regression, tuning $\lambda$ to control the effective dimension of model space.

Many non-parametric regression methods exist, and all perform equally well in one dimension. The real challenge is in the modeling/estimation of functions on multidimensional domains, and generalizations of (1) lead to unparalleled operational convenience and a rich collection of modeling tools.

1.1. Penalized likelihood method. The penalized likelihood method results from an abstraction of (1). To estimate a function of interest $\eta$ on a generic domain $x$ using stochastic data, one may minimize

$$
L(\eta \mid \text { data })+\lambda J(\eta)
$$




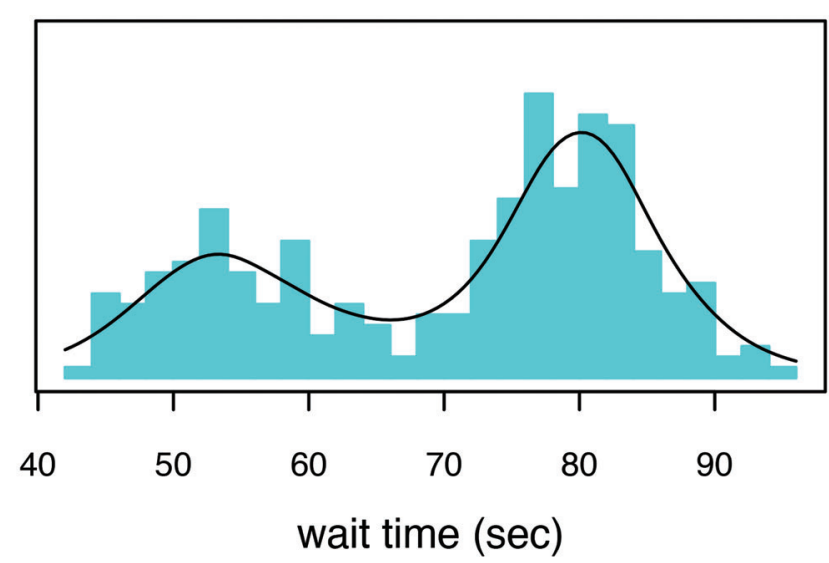

Figure 2. Density estimation.

where $L(\eta \mid$ data) can be taken as the minus log likelihood of the data and $J(\eta)$ is a quadratic functional quantifying the roughness of $\eta$.

In a step towards an abstract $J(\eta)$, Kimeldorf and Wahba [4-6] considered $J(\eta)=\int_{a}^{b}(L \eta)^{2}(x) d x$ on $x=[a, b]$ for some general differential operator $L$, with $J(\eta)=$ $\int_{a}^{b}\left(\eta^{(m)}(x)\right)^{2} d x$ being special cases yielding polynomial smoothing splines. More examples of $X$ and $J(\eta)$ will follow shortly.

A smoothing spline is defined as the solution to a variational problem of the form given in (2). Depending on the configuration, it may or may not reduce to a piecewise polynomial.

The first term in (1) is proportional to the minus log likelihood of the Gaussian regression model stated above. Two more examples of $L(\eta \mid$ data $)$ follow.

Example 1 (Logistic regression). Consider $y_{i} \sim$ $\operatorname{Bin}\left(m_{i}, p_{i}\right)$, where $\log \left\{p_{i} /\left(1-p_{i}\right)\right\}=\eta\left(x_{i}\right)$. One may use $L(\eta)=-\sum_{i}\left\{y_{i} \eta\left(x_{i}\right)-m_{i} \log \left(1+e^{\eta\left(x_{i}\right)}\right)\right\}$ for the estimation of the logit function $\eta(x)$.

Example 2 (Density estimation). Let $x_{i}$ be independent samples from a probability density $p(x)$ on domain $x$. To estimate $p(x)=e^{\eta(x)} / \int_{x} e^{\eta(x)}$, one may use

$$
L(\eta)=-\sum_{i}\left\{\eta\left(x_{i}\right)-\log \int_{x} e^{\eta(x)}\right\}
$$

Example 1 is a special case of non-Gaussian regression. A variant of Example 2 was studied by Silverman [11].

Shown in Figure 2 is an example of one-dimensional density estimation using $J(\eta)=\int_{a}^{b}\left(\eta^{\prime \prime}(x)\right)^{2} d x$; the data are 272 waiting times between eruptions of the Old Faithful geyser, found in the $\mathrm{R}$ data frame faithfu1. Plotted are a cross-validated density estimate (see $\$ 3$ ) using ssden in $\mathrm{R}$ package gss, along with a histogram of the data. This is an instance of unsupervised learning.
The penalized likelihood of (2) is in fact performing constrained maximum likelihood estimation,

$$
\min L(\eta \mid \text { data }) \quad \text { s.t. } J(\eta) \leq \rho,
$$

using the Lagrange method, with $\lambda$ being the Lagrange multiplier; see [3, Theorem 2.12], where $\lambda \propto \rho^{-1}$ is also quantified.

The null space of $J(\eta), \mathcal{N}_{J}=\{\eta: J(\eta)=0\}$, specifies a parametric model for $\eta$. With a $\rho>0$, data-adaptive through the selection of $\lambda$, one allows for $\eta$ to depart from the parametric model.

1.2. Reproducing kernel Hilbert spaces. The minimization of (2) is implicitly in the space $\{\eta: J(\eta)<\infty\}$ or a subspace therein, and function evaluations typically appear in $L(\eta \mid$ data $)$. To facilitate analysis and computation, one needs a metric and a geometry in the function space, and needs the evaluation functional to be continuous.

A reproducing kernel Hilbert space is a Hilbert space $\mathcal{H}$ of functions on a domain $X$ in which the evaluation functional $[x] \eta=\eta(x)$ is continuous, $\forall x \in X, \forall \eta \in \mathcal{H}$. A comprehensive theory of reproducing kernel Hilbert spaces can be found in [1].

By Riesz representation, there exists a reproducing kernel, a non-negative definite bivariate function $R(x, y)$ dual to the inner product $\langle\cdot, \cdot\rangle$ in $\mathcal{H}$, which satisfies $\langle R(x, \cdot), \eta(\cdot)\rangle=\eta(x), \forall x \in \mathcal{X}, \forall \eta \in \mathcal{H}$.

A reproducing kernel Hilbert space can also be generated from its reproducing kernel $R(x, y)$, for which any nonnegative definite function qualifies, as the "column space" $\operatorname{span}\{R(x, \cdot), x \in X\}$. The corresponding inner product may or may not have an explicit expression, however.

For use in (2), one takes $\mathcal{H}=\{\eta: J(\eta)<\infty\}$ equipped with $\langle\cdot, \cdot\rangle=J(\cdot, \cdot)+\tilde{J}(\cdot, \cdot)$, where $J(\cdot, \cdot)$ is the semi-inner product associated with the quadratic functional $J(\eta)$ and $\tilde{J}(\cdot, \cdot)$ is an inner product in the null space $\mathcal{N}_{J}$. One has a tensor-sum decomposition $\mathcal{H}=\mathcal{N}_{J} \oplus \mathcal{H}_{J}$ with $J(\cdot, \cdot)$ being a full inner product in $\mathcal{H}_{J}=\{\eta: J(\eta)<\infty, \tilde{J}(\eta)=0\}$.

Example 3 (Cubic spline). Consider, on $X=[0,1], J(\eta)=$ $\int_{0}^{1}\left(\eta^{\prime \prime}(x)\right)^{2} d x$. For an inner product in $\mathcal{N}_{J}$, take $\tilde{J}(\eta, \eta)=$ $\left(\int_{0}^{1} \eta(x) d x\right)^{2}+\left(\int_{0}^{1} \eta^{\prime}(x) d x\right)^{2}$. It follows that $\int_{0}^{1} \eta(x) d x=$ $\int_{0}^{1} \eta^{\prime}(x) d x=0, \forall \eta \in \mathcal{H}_{J}$.

The reproducing kernel in $\mathcal{H}_{J}$ is known to be $R_{J}(x, y)=$ $k_{2}(x) k_{2}(y)-k_{4}(x-y)$, where $k_{\nu}=B_{\nu} / \nu$ ! are scaled Bernoulli polynomials. One may further decompose $\mathcal{N}_{J}=\{1\} \oplus$ $\left\{k_{1}(x)\right\}$ for $k_{1}(x)=x-0.5$, with the respective reproducing kernels given by $R_{00}(x, y)=1$ and $R_{01}(x, y)=k_{1}(x) k_{1}(y)$.

Facts concerning tensor-sum decompositions of reproducing kernel Hilbert spaces can be found in [3, Theorem 2.5], and technical details of Example 3 are in Craven and Wahba [2]. 
The theory of reproducing kernel Hilbert spaces provides an abstract mathematical framework encompassing a great variety of problems. The abstract setting allows many important issues, such as the computation and the asymptotic convergence of the minimizers of (2), be treated in a unified fashion. As summarized in Grace's 1990 monograph [16], much of her work up to that date, from approximation theory to spline smoothing, fit under the general framework.

1.3. Tensor product splines. A statistical model should be interpretable, which distinguishes it from mere function approximation or some black-box predictor/classifier. Two main challenges in the non-parametric modeling of multivariate data are weak interpretability and the curse of dimensionality, which might be alleviated via a hierarchical structure of the functional ANOVA decomposition.

Functional ANOVA decomposition. Consider a bivariate function $\eta(x)=\eta\left(x_{\langle 1\rangle}, x_{\langle 2\rangle}\right)$ on $x=x_{1} \times x_{2}$; subscripts in brackets denote coordinates of a point on a multidimensional domain while ordinary subscripts are reserved for multiple points. One may write

$$
\begin{aligned}
\eta(x)= & \left(I-A_{1}+A_{1}\right)\left(I-A_{2}+A_{2}\right) \eta \\
= & A_{1} A_{2} \eta+\left(I-A_{1}\right) A_{2} \eta \\
& +A_{1}\left(I-A_{2}\right) \eta+\left(I-A_{1}\right)\left(I-A_{2}\right) \eta \\
= & \eta_{\emptyset}+\eta_{1}\left(x_{\langle 1\rangle}\right)+\eta_{2}\left(x_{\langle 2\rangle}\right)+\eta_{12}\left(x_{\langle 1\rangle}, x_{\langle 2\rangle}\right),
\end{aligned}
$$

where $I$ is the identity operator, $A_{1}, A_{2}$ are averaging operators acting on arguments $x_{\langle 1\rangle}, x_{\langle 2\rangle}$, respectively, that satisfy $A 1=1, \eta_{1}, \eta_{2}$ are main effects, and $\eta_{12}$ is the interaction, satisfying $A_{1} \eta_{1}=A_{1} \eta_{12}=0, A_{2} \eta_{2}=A_{2} \eta_{12}=0$. Similar constructions in more than two dimensions are straightforward.

Examples of averaging operators include $A \eta=$ $\int_{a}^{b} \eta(x) d x /(b-a)$ on $[a, b], A \eta=\sum_{i=1}^{m} \eta\left(x_{i}\right) / m$ on any domain; averaging operators on different axes are independent of each other.

For $x_{1} \times x_{2}$ discrete, $\eta\left(x_{\langle 1\rangle}, x_{\langle 2\rangle}\right)$ is a matrix of treatment means usually denoted by $\mu_{i j}$ in a standard two-way ANOVA model, with (4) in the form

$$
\begin{aligned}
\mu_{i j}= & \mu_{. .}+\left(\mu_{i .}-\mu_{. .}\right) \\
& +\left(\mu_{. j}-\mu_{. .}\right)+\left(\mu_{i j}-\mu_{i .}-\mu_{. j}+\mu_{. .}\right) \\
= & \mu+\alpha_{i}+\beta_{j}+(\alpha \beta)_{i j},
\end{aligned}
$$

where $\mu_{i}=\sum_{j} c_{j} \mu_{i j}$ for $\sum_{j} c_{j}=1, \mu_{. j}=\sum_{i} d_{i} \mu_{i j}$ for $\sum_{i} d_{i}=1$, and $\mu_{. .}=\sum_{i, j} c_{j} d_{i} \mu_{i j}$.

Selective term elimination in functional ANOVA decompositions helps to combat the curse of dimensionality in estimation and facilitates the interpretation of the analysis. For example, the so-called additive models, those containing only main effects, are easier to estimate and interpret than ones involving interactions. As with classical ANOVA models on discrete domains, the inclusion of higher-order interactions are to be avoided in practice.

For $\eta$ a log density, absence of selected interactions may imply (conditional) independence structures among random variables. Taking random variables $(X, Y, Z)$ on domain $x \times y \times z$, say, a log density of the form $\eta=$ $\eta_{\emptyset}+\eta_{x}+\eta_{y}+\eta_{z}+\eta_{x y}+\eta_{x z}$ implies the conditional independence of $Y$ and $Z$ given $X$, or $Y \perp Z \mid X$, where the notation for ANOVA terms of $\eta(x, y, z)$ parallels that in (4).

Tensor product spaces. For the estimation of $\eta$ on product domains via (2), functional ANOVA decompositions can be built in through the construction of tensor product reproducing kernel Hilbert spaces.

Theorem 1. Consider $x=x_{1} \times x_{2}$ and $x, y \in x$. For $R_{\langle 1\rangle}\left(x_{\langle 1\rangle}, y_{\langle 1\rangle}\right)$ non-negative definite on $x_{1}$ and $R_{\langle 2\rangle}\left(x_{\langle 2\rangle}, y_{\langle 2\rangle}\right)$ nonnegative definite on $x_{2}, R(x, y)=R_{\langle 1\rangle}\left(x_{\langle 1\rangle}, y_{\langle 1\rangle}\right) R_{\langle 2\rangle}\left(x_{\langle 2\rangle}, y_{\langle 2\rangle}\right)$ is non-negative definite on $x=x_{1} \times x_{2}$.

To construct a reproducing kernel Hilbert space, it suffices to specify a reproducing kernel. The following example illustrates a construction on $x=[0,1]^{2}$ using the results of Example 3.

Example 4 (Tensor-product cubic spline). The space $\mathcal{H}=$ $\left\{\eta: \int_{0}^{1}\left(\eta^{\prime \prime}(x)\right)^{2} d x<\infty\right\}$ is decomposed as $\{1\} \oplus\left\{k_{1}(x)\right\} \oplus$ $\mathcal{H}_{J}$ in Example 3. We rewrite the decomposition as $\mathcal{H}_{00} \oplus$ $\mathcal{H}_{01} \oplus \mathcal{H}_{1}$, and denote the respective reproducing kernels as $R_{00}, R_{01}$, and $R_{1}$. A one-way ANOVA decomposition is built in, with $\eta \in \mathcal{H}_{01} \oplus \mathcal{H}_{1}$ satisfying $A \eta=\int_{0}^{1} \eta(x) d x=0$.

Using these reproducing kernels on the two axes and taking products as in Theorem 1, one has nine reproducing kernels on $[0,1]^{2}$, each yielding a tensor product space, as laid out below:

$$
\begin{array}{ccc}
\mathcal{H}_{00,00} & \mathcal{H}_{00,01} & \mathcal{H}_{00,1} \\
\mathcal{H}_{01,00} & \mathcal{H}_{01,01} & \mathcal{H}_{01,1} \\
\mathcal{H}_{1,00} & \mathcal{H}_{1,01} & \mathcal{H}_{1,1}
\end{array}
$$

The ANOVA decomposition of (4) is built in, with $\eta_{\emptyset} \in$ $\mathcal{H}_{00,00}, \eta_{1} \in \mathcal{H}_{01,00} \oplus \mathcal{H}_{1,00}$, etc.

Pasting these spaces together via tensor-sum, one has what is needed for use in (2). The four spaces on the upperleft corner are of one dimension each, and can be lumped into $\mathcal{N}_{J}$. The remaining five spaces can be put together as $\mathcal{H}_{J}$ with a reproducing kernel

$$
\begin{aligned}
R_{J}= & \theta_{00,1} R_{00,1}+\theta_{1,00} R_{1,00} \\
& +\theta_{01,1} R_{01,1}+\theta_{1,01} R_{1,01}+\theta_{1,1} R_{1,1},
\end{aligned}
$$

where the $\theta^{\prime}$ s are extra smoothing parameters adjusting the relative contribution of each component to the overall roughness measure.

To enforce an additive model, one removes $\mathcal{H}_{01,01}$ from $\mathcal{N}_{J}$ and sets $\theta_{1,01}=\theta_{01,1}=\theta_{1,1}=0$.

The construction described in Example 4 is at an abstract level, requiring little specifics of cubic splines on 

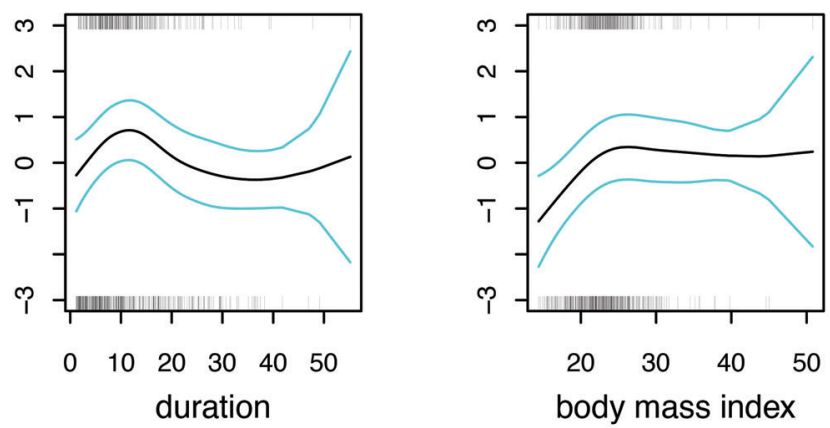

Figure 3. Additive logistic regression.

$[0,1]$. All one needs are reproducing kernels on marginal domains. Functional ANOVA decomposition follows trivially from one-way ANOVA decompositions on marginal domains.

Denote by $\mathcal{H}_{\beta}$ the component spaces of $\mathcal{H}_{J}$ in Example 4 and write $R_{\beta}, J_{\beta}$ as the respective reproducing kernels and the associated square norms. With $R_{J}=\sum_{\beta} \theta_{\beta} R_{\beta}$, the corresponding square norm in $\mathcal{H}_{J}$ is given by $J(\eta)=$ $\sum_{\beta} \theta_{\beta}^{-1} J_{\beta}(\eta)$, to be used in (2).

As an illustration, we fit a logistic regression model of Example 1 to the wesdr data found in R package gss, concerning the progression of diabetic retinopathy. One has $x=\left(x_{\langle 1\rangle}, x_{\langle 2\rangle}, x_{\langle 3\rangle}\right) \in R^{3}$ and a binary $y \sim \operatorname{Bin}(1, p(x))$, and the full model would include three main effects, three twoway interactions, and a three-way interaction. After some exploration, the interaction terms are found to be negligible so an additive model appears adequate, and one of the main effects is actually linear. We finally fit a model

$$
\eta(x)=\beta_{0}+\beta_{1} x_{\langle 1\rangle}+\eta_{2}\left(x_{\langle 2\rangle}\right)+\eta_{3}\left(x_{\langle 3\rangle}\right),
$$

where $\eta=\log p /(1-p)$; this is actually a partial spline model discussed at the end of this section. Plotted in Figure 3 are estimated $\eta_{2}$ and $\eta_{3}$ along with their Bayesian confidence intervals (see $\$ 4$ ). The rugs on the ceiling and the floor mark the observed $y=1$ and $y=0$, respectively, though $p(x)$ does not depend on $x_{\langle 2\rangle}$ or $x_{\langle 3\rangle}$ alone; the confidence intervals do get wider where data are sparse. The analysis was done using the gssanova facilities in package gss. Further details can be found in [3, \$5.5.3].

Grace's signature was all over the tensor product spline technique, from the inception of the idea to the ensuing rigorous developments, involving several of her students including your authors; see, e.g., [16, Chap. 10] and [18].

1.4. More splines. Real intervals are the most encountered domains in practice, which can be mapped onto $[0,1]$, and the cubic spline on $[0,1]$ with $J(\eta)=$ $\int_{0}^{1}\left(\eta^{\prime \prime}(x)\right)^{2} d x$ is the commonly used configuration for data smoothing in the setting. On domains other than real intervals or to accommodate various special needs, alternatives to cubic splines are sometimes called for.
We now present a variety of configurations tuned to various situations, which may be used directly in (2) on the respective designated domains, or be used as building blocks to construct tensor product splines.

Periodic splines. To accommodate recurring patterns such as circadian rhythms or seasonal effects, one may consider only periodic functions on a real interval. Mapping the interval onto $[0,1]$ and setting $J(\eta)=\int_{0}^{1}\left(\eta^{\prime \prime}(x)\right)^{2} d x$ for $\eta$ periodic, one has $\mathcal{H}=\{1\} \oplus \mathcal{H}_{J}$, with component space reproducing kernels $R_{0}(x, y)=1$ and $R_{J}(x, y)=-k_{4}(x-y)$; see Craven and Wahba [2].

L-splines. On $[0,1]$, one may configure an L-spline by setting $J(\eta)=\int_{0}^{1}(L \eta)^{2}(x) h(x) d x$, where $L$ is a general differential operator and $h(x)>0$ is a weight function. With $L=D^{m}$ and $h(x)=1$, it reduces to a polynomial spline; $m=2$ yields the cubic spline.

When the null space $\mathcal{N}_{L}=\{\eta: L \eta=0\}$ forms a more desirable parametric model than lower-order (say linear) polynomials, the corresponding L-spline is preferred over a polynomial spline.

Example 5 (Exponential spline). For $\theta>0$, set $L=$ $D(D-\theta)$ and $h(x)=e^{-3 \theta x}$. The null space $\mathcal{N}_{L}=\{\eta$ : $\left.\eta(x)=\beta_{0}+\beta_{1} e^{\theta x}\right\}$ makes a reasonable model for a growth curve.

Transforming $x$ by $\tilde{x}=\left(e^{\theta x}-1\right) / \theta$, it can be shown that $\int_{0}^{1}(L \eta)^{2}(x) h(x) d x=\int_{0}^{a}\left(d^{2} \eta / d \tilde{x}^{2}\right)^{2} d \tilde{x}$, where $a=\left(e^{\theta}-1\right) / \theta$, yielding a cubic spline in $\tilde{x}$.

As noted earlier, L-splines were studied by Kimeldorf and Wahba [4-6], showcasing an abstract $J(\eta)$.

Thin-plate splines. On $X=R^{d}$, for $2 m>d$, one may use

$$
\begin{aligned}
J(\eta)= & \sum_{\alpha_{1}+\cdots+\alpha_{d}=m} \frac{m !}{\alpha_{1} ! \cdots \alpha_{d} !} \\
& \int \cdots \int\left(\frac{\partial^{m} \eta}{\partial x_{\langle 1\rangle}^{\alpha_{1}} \cdots \partial x_{\langle d\rangle}^{\alpha_{d}}}\right)^{2} d x_{\langle 1\rangle} \cdots d x_{\langle d\rangle},
\end{aligned}
$$

which is invariant to coordinate rotation and shift. For $d=1, m=2$, this reduces to a cubic spline. For $d=2$, $m=2$, one has

$$
J(\eta)=\iint\left(\left(\eta_{1,1}^{(2)}\right)^{2}+2\left(\eta_{1,2}^{(2)}\right)^{2}+\left(\eta_{2,2}^{(2)}\right)^{2}\right) d x_{\langle 1\rangle} d x_{\langle 2\rangle},
$$

where $\eta_{1,2}^{(2)}=\partial^{2} \eta / \partial x_{\langle 1\rangle} \partial x_{\langle 2\rangle}$, etc. The reproducing kernels are rather involved to specify for $d>1$; technical details and references can be found in $[3, \S 4.3]$ and $[16, \$ 2.4]$.

Thin-plate splines provide natural analytical tools for spatial smoothing and geographic mapping, where directional decompositions such as latitude/longitude effects may not make sense. When used as marginal domains in tensor product splines, the mathematically multidimensional $R^{d}$ acts as an inseparable entity, contributing one logical dimension. 
Some early derivations and meteorology applications of thin-plate splines can be found in Wahba and Wendelberger [19].

Spherical splines. To estimate functions on small geographic areas, one may use thin-plate splines on $R^{2}$, but surface curvature cannot be ignored on larger geographic regions or for global mapping. The spherical splines of Wahba [13] were developed just for this purpose.

On the unit sphere $\mathcal{S}$, consider the Laplace-Beltrami operator

$$
\Delta=\frac{1}{\sin ^{2} \theta} \frac{\partial^{2}}{\partial \phi^{2}}+\frac{1}{\sin \theta} \frac{\partial}{\partial \theta}\left(\sin \theta \frac{\partial}{\partial \theta}\right)
$$

and set $J(\eta)=\int_{0}^{2 \pi} \int_{0}^{\pi}(\Delta \eta)^{2} \sin \theta d \theta d \phi$, where $\theta \in[0, \pi]$ is latitude and $\phi \in[0,2 \pi]$ is longitude. Such a $J(\eta)$ is invariant under coordinate rotation. The null space is $\mathcal{N}_{J}=\{1\}$, and the reproducing kernel associated with $J(\eta)$ is available as an infinite sum involving spherical harmonics.

Technical details rely heavily on the mathematics of spherical harmonics, as outlined in [3, \$4.4] and $[16, \S 2.2]$. Similar to thin-plate splines, the unit sphere $\mathcal{S}$ contributes one logical dimension in tensor product splines.

Discrete splines. Consider a discrete domain $\{1, \ldots, K\}$, on which a function is a vector, and a bivariate function a matrix.

When the domain is nominal, or the labeling of the elements is arbitrary, a natural choice is

$$
J(\eta)=\sum_{x=1}^{K}(\eta(x)-\bar{\eta})^{2},
$$

where $\bar{\eta}=K^{-1} \sum_{x=1}^{K} \eta(x)$.

When the domain is ordinal, one may use $J(\eta)=$ $\sum_{x=2}^{K}(\eta(x)-\eta(x-1))^{2}$.

Write $J(\eta)=\eta^{T} J \eta$, where $\eta=(\eta(1), \ldots, \eta(K))^{T}$ and the matrix $J$ is non-negative definite. $J(\eta)$ is a full square norm in $\mathcal{H}_{J}=\{\eta: \eta=J \mathbf{c}\}$, the column space of $J$, with reproducing kernel $R_{J}=J^{+}$, the Moore-Penrose inverse of $J$. See $[3, \$ 2.2]$.

Standing alone, penalized estimation on discrete domains is known as shrinkage having its own literature. The purpose of this discussion is to configure building blocks for use in tensor product splines when some of the variables are discrete.

Partial splines. As evident by now, estimation via (2) along with the functional ANOVA structure provides a rich family of non-parametric statistical models. When knowledge is sufficient to justify parametric forms for part of the model, one has semiparametric models [20, Chap. 8]. A special case of such models can be written as $y_{i}=\mathbf{z}_{i}^{T} \beta+\eta\left(x_{i}\right)+$ $\epsilon_{i}$ in a Gaussian regression setting, where $\mathbf{z}_{i}^{T} \beta$ comprises partial terms, and one may estimate $\beta$ and $\eta(x)$ jointly via the minimization of

$$
\sum_{i}\left(y_{i}-\mathbf{z}_{i}^{T} \beta-\eta\left(x_{i}\right)\right)^{2}+\lambda J(\eta)
$$

The partial terms can be readily accommodated in (2) via trivial manipulations of the $L(\eta)$ term. Caution must be exercised in practice, however, as the partial terms $\mathbf{z}_{i}^{T} \beta$ may not be identifiable from $\eta\left(x_{i}\right)$ using the available data.

Some theoretical analysis of partial splines can be found in [16, Chap. 6].

\section{Representation}

The function space $\mathcal{H}$ is of infinite dimension in general. To numerically calculate the minimizer of (2) in $\mathcal{H}$, one needs adequate explicit expression for $\eta \in \mathcal{H}$.

2.1. Finite-dimensional solution. On domain $X$, consider the minimization of

$$
\frac{1}{n} \sum_{i=1}^{n}\left(y_{i}-\eta\left(x_{i}\right)\right)^{2}+\lambda J(\eta)
$$

over $\eta \in \mathcal{H}=\mathcal{N}_{J} \oplus \mathcal{H}_{J}$, where $\mathcal{N}_{J} \subseteq\{\eta: J(\eta)=0\}$ is of finite dimension and $J(\eta)=J(\eta, \eta)$ is the square norm in $\mathcal{H}_{J}$ dual to reproducing kernel $R_{J}(x, y) ; J\left(R_{J}(x, \cdot), \eta(\cdot)\right)=$ $\eta(x), \forall \eta \in \mathcal{H}_{J}$.

Theorem 2. The minimizer of (5) has a finite-dimensional representation

$$
\eta(x)=\sum_{\nu=1}^{m} d_{\nu} \phi_{\nu}(x)+\sum_{i=1}^{n} c_{i} R_{J}\left(x_{i}, x\right),
$$

where $\left\{\phi_{\nu}\right\}_{\nu=1}^{m}$ is a basis of $\mathcal{N}_{J}$.

This cornerstone result is due to Kimeldorf and Wahba [6], presented in an L-spline setting, the most abstract form known at the time.

The proof of the theorem is via a simple, clever geometric argument. Functions in $\mathcal{H}$ can be expressed in a form

$$
\eta(x)=\sum_{\nu=1}^{m} d_{\nu} \phi_{\nu}(x)+\sum_{i=1}^{n} c_{i} R_{J}\left(x_{i}, x\right)+\rho(x),
$$

where $\rho(x) \in \mathcal{H}_{J} \ominus \operatorname{span}\left\{R_{J}\left(x_{i}, \cdot\right), i=1, \ldots, n\right\}$. Plugging (7) into (5), one has

$$
(\mathbf{y}-S \mathbf{d}-Q \mathbf{c})^{T}(\mathbf{y}-S \mathbf{d}-Q \mathbf{c})+n \lambda \mathbf{c}^{T} Q \mathbf{c}+n \lambda J(\rho),
$$

where $\mathbf{y}=\left(y_{1}, \ldots, y_{n}\right)^{T}, S$ is $n \times m$ with the $(i, v)$ th entry $\phi_{\nu}\left(x_{i}\right)$, and $Q$ is $n \times n$ with the $(i, j)$ th entry $R_{J}\left(x_{i}, x_{j}\right)=$ $J\left(R_{J}\left(x_{i}, \cdot\right), R_{J}\left(x_{j}, \cdot\right)\right)$; note that $\rho\left(x_{i}\right)=J\left(R_{J}\left(x_{i}, \cdot\right), \rho(\cdot)\right)=0$.

Clearly, $\rho(x)=0$ for the minimizer of (5), yielding (6). The result holds in general when $L(\eta)$ in (2) depends on $\eta$ only through function evaluations $\eta\left(x_{i}\right)$.

The abstract setting allows generic algorithms to be developed and implemented, and the numerical calculation requires only a basis of $\mathcal{N}_{J}$ and the reproducing kernel $R_{J}$ of $\mathcal{H}_{J}$; specifically, an explicit form of $J(\eta)$ is not needed. 
For tensor product splines, $R_{J}=\sum_{\beta} \theta_{\beta} R_{\beta}$, and the solution is in the form

$$
\eta(x)=\sum_{\nu=1}^{m} d_{\nu} \phi_{\nu}(x)+\sum_{i=1}^{n} c_{i}\left(\sum_{\beta} \theta_{\beta} R_{\beta}\left(x_{i}, x\right)\right) .
$$

ANOVA terms are readily available in partial sums of $d_{\nu} \phi_{\nu}(x)$ and $\theta_{\beta} \sum_{i=1}^{n} c_{i} R_{\beta}\left(x_{i}, x\right)$; note the same $c_{i}$ 's used in all penalized terms.

2.2. Asymptotic convergence. Denote by $\eta_{\lambda}$ the minimizer of (5) in $\mathcal{H}$, and let $f(x)$ be the limiting density of $\left\{x_{i}\right\}_{i=1}^{n}$ on $X$. Under conditions, one can show that as $n \rightarrow \infty, \lambda \rightarrow 0$,

$$
(V+\lambda J)\left(\eta_{\lambda}-\eta\right)=O_{p}\left(\lambda^{p}+n^{-1} \lambda^{-1 / r}\right),
$$

where $V(\eta)=\int_{x} \eta^{2}(x) f(x) d x, p \in[1,2]$ depending on how smooth the true $\eta$ is, and $r$ codes the regulating power of $J(\eta)$. For the cubic splines on $[0,1], r=4, p=1$ if $\eta(x)$ "barely" satisfies $\int_{0}^{1}\left(\eta^{\prime \prime}(x)\right)^{2} d x<\infty$, and $p=2$ if $\int_{0}^{1}\left(\eta^{(4)}(x)\right)^{2} d x<\infty$.

$V\left(\eta_{\lambda}-\eta\right) \approx n^{-1} \sum_{i=1}^{n}\left(\eta_{\lambda}\left(x_{i}\right)-\eta\left(x_{i}\right)\right)^{2}$, the mean square error at data points. The optimal convergence rate $O_{p}\left(n^{-p r /(p r+1)}\right)$ is achieved at $\lambda \asymp n^{-r /(p r+1)}$. For $p=2$, $r=4$, the rate would be $O_{p}\left(n^{-8 / 9}\right)$, not too far from the $O_{p}\left(n^{-1}\right)$ rate for parametric models. The minimizer $\eta_{\lambda}$ of (5) can indeed be a good estimate of $\eta$, assuming $\lambda$ can be selected properly.

Smoothing via $J(\eta) \leq \rho \propto \lambda^{-1}$ is like applying a lowpass filter, and the effective "active" subspace should be of dimension in the ballpark of $O\left(\lambda^{-1 / r}\right)$. The solution expression in (6) asserts that this "active" subspace is entirely contained in $\mathcal{N}_{J} \oplus \operatorname{span}\left\{R_{J}\left(x_{i}, \cdot\right), i=1, \ldots, n\right\}$, but there likely remain further redundancies among $R_{J}\left(x_{i}, \cdot\right)$ 's.

In fact, the same convergence rate as in (10) also holds for the minimizer $\eta_{\lambda}^{*}$ of (5) in a space

$$
\mathcal{H}^{*}=\mathcal{N}_{J} \oplus \operatorname{span}\left\{R_{J}\left(z_{j}, \cdot\right), j=1, \ldots, q\right\},
$$

where $q \lambda^{2 / r} \rightarrow \infty$ and $\left\{z_{j}\right\}_{j=1}^{q}$ is a random subset of $\left\{x_{i}\right\}_{i=1}^{n}$. Note the role $f(x)$ plays in the definition of $V(\eta)$, and a random selection of $\left\{z_{j}\right\}$ mimics $f(x)$. It suffices to use $q \asymp \lambda^{-2 / r} n^{\epsilon}, \forall \epsilon>0$, and for $\lambda \asymp n^{-r /(p r+1)}$, this leads to $q \asymp n^{2 /(p r+1)+\epsilon}$.

The $q R_{J}\left(z_{j}, \cdot\right)^{\prime} \mathrm{s}, q \asymp \lambda^{-2 / r} n^{\epsilon}$, provide sufficient coverage of the "active" subspace of dimension $O\left(\lambda^{-1 / r}\right)$. In principle, one might be able to achieve the same effect with a smaller set of bases via delicate selection in specific settings, but the worry-free random selection in (11) is justified in an abstract setting. Computation using $q$ bases is of order $O\left(n q^{2}\right)$.

As an estimate of $\eta, \eta_{\lambda}^{*}$ is as efficient asymptotically performance-wise, thus is called an efficient approximation of $\eta_{\lambda}$.
Similar results hold for other configurations of $L(\eta)$ in (2), but the definition of $V(\eta)$ naturally varies with the settings. See [3, Chap. 9] for detailed asymptotic analysis in a variety of stochastic settings, using techniques originated in [11].

When $L(\eta)$ depends on $\eta$ through more than a finite number of function evaluations, such as with the density estimation of Example 2, the minimizer $\eta_{\lambda}$ in $\mathcal{H}$ may not be computable, but the efficient approximation $\eta_{\lambda}^{*}$ makes the method practically applicable.

Functions in $\mathcal{H}^{*}$ of (11) have an expression

$$
\eta(x)=\sum_{\nu=1}^{m} d_{\nu} \phi_{\nu}(x)+\sum_{j=1}^{q} c_{j} R_{J}\left(z_{j}, x\right),
$$

with (6) as a special case at $q=n$.

\section{Smoothing Parameter Selection}

The convergence rates confirm that the method is capable of delivering good estimates, and the finite-dimensional representation makes computation possible. Varying the $\lambda$ in front of $J(\eta)$ and the possible $\theta^{\prime}$ s hidden in $J(\eta)$ as with tensor product splines, one has available a family of estimates, from which we hope to pick well-performing members.

The practical success of (2) hinges on the proper selection of smoothing parameters. In what follows, we shall use $\lambda$ to denote both the $\lambda$ in front of $J(\eta)$ and the possible $\theta$ 's hidden therein. The minimizer of (2) to be calculated, likely in $\mathcal{H}^{*}$, is denoted by $\eta_{\lambda}$.

3.1. Performance measures. The purpose of $\lambda$ selection is to pick well-performing estimates, but one first needs to define what good performance means. Performance measures naturally vary with stochastic settings.

Gaussian regression. For Gaussian regression via (5), a natural performance measure is the mean square error over $\left\{x_{i}\right\}$

$$
L(\lambda)=\frac{1}{n} \sum_{i=1}^{n}\left(\eta_{\lambda}\left(x_{i}\right)-\eta\left(x_{i}\right)\right)^{2} .
$$

$L(\lambda)$ in (12) is a statistical loss, with minimum at say $\lambda_{o}$, the data-specific optimal choice. The optimal $\lambda_{o}$ is beyond reach in practice, as $\eta(x)$ is unknown except in simulations. Density estimation. For another stochastic setting, consider the estimation of probability density $p(x)=$ $e^{\eta(x)} / \int_{x} e^{\eta(x)} d x$ on $X$ via the minimization of

$$
-\frac{1}{n} \sum_{i=1}^{n} \eta\left(x_{i}\right)+\log \int_{x} e^{\eta(x)} d x+\frac{\lambda}{2} J(\eta) .
$$

A natural performance measure is the Kullback-Leibler divergence,

$$
L(\lambda)=\mu_{\eta}\left(\eta-\eta_{\lambda}\right)-\log \int_{x} e^{\eta(x)} d x+\log \int_{x} e^{\eta_{\lambda}(x)} d x,
$$

where $\mu_{g}(h)=\int_{x} h(x) e^{g(x)} d x / \int_{x} e^{g(x)} d x$. 
3.2. Cross validation. The statistical loss $L(\lambda)$ involves the unknown $\eta$ and the candidate estimate $\eta_{\lambda}$. In many stochastic settings, one may decompose

$$
L(\lambda)=A\left(\eta_{\lambda}\right)+B\left(\eta, \eta_{\lambda}\right)+C(\eta)
$$

where $A\left(\eta_{\lambda}\right)$ can be computed, $C(\eta)$ can be dropped as it does not involve $\lambda$, and $B\left(\eta, \eta_{\lambda}\right)$ is to be estimated via cross validation, yielding some $V(\lambda)=A\left(\eta_{\lambda}\right)+\hat{B}\left(\eta, \eta_{\lambda}\right)$ for use as the selection tool.

In general, one is looking for some computable $V(\lambda)$ that roughly parallels $L(\lambda)$, so the minimizer of $V(\lambda)$ may have a good chance to deliver near optimal performance. Density estimation. An example of the structure described above is the Kullback-Leibler divergence in (13), where $A\left(\eta_{\lambda}\right)=\log \int_{x} e^{\eta_{\lambda}(x)} d x$ and $B\left(\eta, \eta_{\lambda}\right)=-\mu_{\eta}\left(\eta_{\lambda}\right)$. One may estimate $\mu_{\eta}\left(\eta_{\lambda}\right)$ using a cross-validated sample mean, $n^{-1} \sum_{i=1}^{n} \eta_{\lambda}^{[i]}\left(x_{i}\right)$, with $\eta_{\lambda}^{[i]}$ minimizing

$$
-\frac{1}{n-1} \sum_{j \neq i} \eta\left(x_{j}\right)+\log \int_{x} e^{\eta(x)} d x+\frac{\lambda}{2} J(\eta) .
$$

Gaussian regression. For Gaussian regression via (5), an ordinary cross validation score is given by

$$
V_{0}(\lambda)=\frac{1}{n} \sum_{i=1}^{n}\left(y_{i}-\hat{y}_{i(i)}\right)^{2},
$$

where $\hat{y}_{i(i)}=\eta_{\lambda}^{[i]}\left(x_{i}\right)$ for $\eta_{\lambda}^{[i]}$ minimizing

$$
\frac{1}{n} \sum_{j \neq i}\left(y_{j}-\eta\left(x_{j}\right)\right)^{2}+\lambda J(\eta) .
$$

Write $\hat{y}_{i}=\eta_{\lambda}\left(x_{i}\right)$ and $\hat{\mathbf{y}}=A(\lambda) \mathbf{y}$, where $A(\lambda)$ is known as the smoothing matrix. It can be shown that $y_{i}-\hat{y}_{i(i)}=$ $\left(y_{i}-\hat{y}_{i}\right) /\left(1-a_{i, i}\right)$, where $a_{i, i}$ is the $(i, i)$ th entry of $A(\lambda)$, so

$$
V_{0}(\lambda)=\frac{1}{n} \sum_{i=1}^{n} \frac{\left(y_{i}-\hat{y}_{i}\right)^{2}}{\left(1-a_{i, i}\right)^{2}}
$$

An invariance argument suggests replacing $a_{i, i}$ 's by their average value $\operatorname{tr} A(\lambda) / n$, yielding the renowned generalized cross validation (GCV) score of Craven and Wahba [2],

$$
V(\lambda)=\frac{n^{-1} \mathbf{y}^{T}(I-A(\lambda))^{2} \mathbf{y}}{\left\{n^{-1} \operatorname{tr}(I-A(\lambda))\right\}^{2}} .
$$

In standard multiple regression, the score in (14) is the familiar PRESS statistic for model selection, where $J(\eta)$ is irrelevant and the continuous scale $\lambda$ is replaced by a discrete set of candidate models.

3.3. Optimality of cross validation. We first reiterate some simple logic. There are two functions of $\lambda$ involved here, the performance measure $L(\lambda)$, and the selection score $V(\lambda)$. The minimizer $\lambda_{o}$ of $L(\lambda)$ defines the dataspecific optimal choice, the best one can hope to do given the data. The minimizer $\lambda_{v}$ of $V(\lambda)$ is the practical selection.

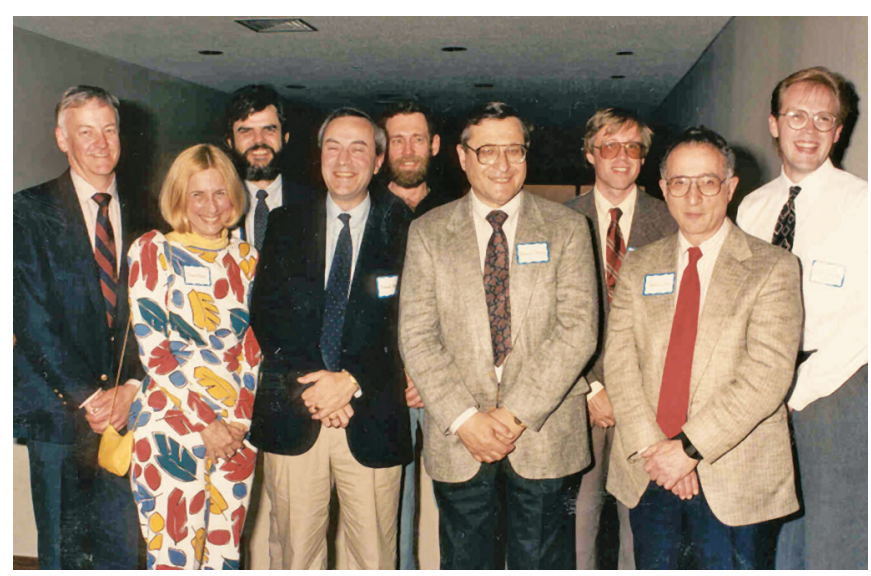

Figure 4. Manny Parzen's 60th birthday party, 1989. From left: Don Ylvisaker, Grace Wahba, Joe Newton, Marcello Pagano, Randy Eubank, Manny Parzen, Will Alexander, Marvin Zelen, Scott Grimshaw.

Once again, $\lambda_{v}$ is not optimal by definition, but may deliver near optimal performance. $V(\lambda)$ is a selection tool, not a performance measure.

To explore the effectiveness of a cross validation score, one may conduct simulation studies, where one knows $\eta$ so can compute $L(\lambda)$ and identify $\lambda_{o}$. If the ratio $L\left(\lambda_{o}\right) / L\left(\lambda_{v}\right)$ is frequently scattered near one, then $V(\lambda)$ is likely a winner. Note that the proximity of $\lambda_{o}$ and $\lambda_{v}$ is meaningless for the purpose, as the bottom of $L(\lambda)$ may be flat or may be steep.

For technical analysis, suggestive results for various cross validation scores in regression settings exist in the form of $V(\lambda)-L(\lambda)-K=o_{p}(L(\lambda))$, where $K$ is some random quantity not involving $\lambda[3, \S \S 6.2 .3,6.3 .3]$; such results fall far short of justifying the use of $V(\lambda)$, however, as the behaviors of the random $\lambda_{o}$ and $\lambda_{v}$ are not accounted for.

The only rigorous theoretical justification known to date was by Ker-Chau Li [8], who showed that the use of the GCV score $V(\lambda)$ in (15) is optimal for the minimization of $L(\lambda)$ in (12), in the sense that $L\left(\lambda_{v}\right) / L\left(\lambda_{o}\right)=1+o_{p}(1)$ as $n \rightarrow \infty$.

\section{Bayes Model}

Consider $y_{i}=\eta\left(x_{i}\right)+\epsilon_{i}, \epsilon_{i} \sim N\left(0, \sigma^{2}\right)$ independent, $x_{i} \in$ $\{1, \ldots, K\}$, and assume $\eta=\mu \mathbf{1}+\alpha$, where $\mu \mathbf{1}$ is fixed effect and $\alpha \sim N\left(\mathbf{0}, b\left(I-\mathbf{1 1}^{T} / K\right)\right)$ is random effect. The posterior mean of $\eta$ is given by the minimizer of

$$
\frac{1}{\sigma^{2}} \sum_{i=1}^{n}\left(y_{i}-\eta\left(x_{i}\right)\right)^{2}+\frac{1}{b} \sum_{x=1}^{K}(\eta(x)-\bar{\eta})^{2} ;
$$

this is discrete spline with $J(\eta)=\sum_{x=1}^{K}(\eta(x)-\bar{\eta})^{2}$ and $\lambda=\sigma^{2} / n b$.

In general, let $\eta(x)=\eta_{0}(x)+\eta_{1}(x)$, where $\eta_{0}(x)$ is diffuse in $\mathcal{N}_{J}$ (fixed effect) and $\eta_{1}(x)$ is a mean zero 


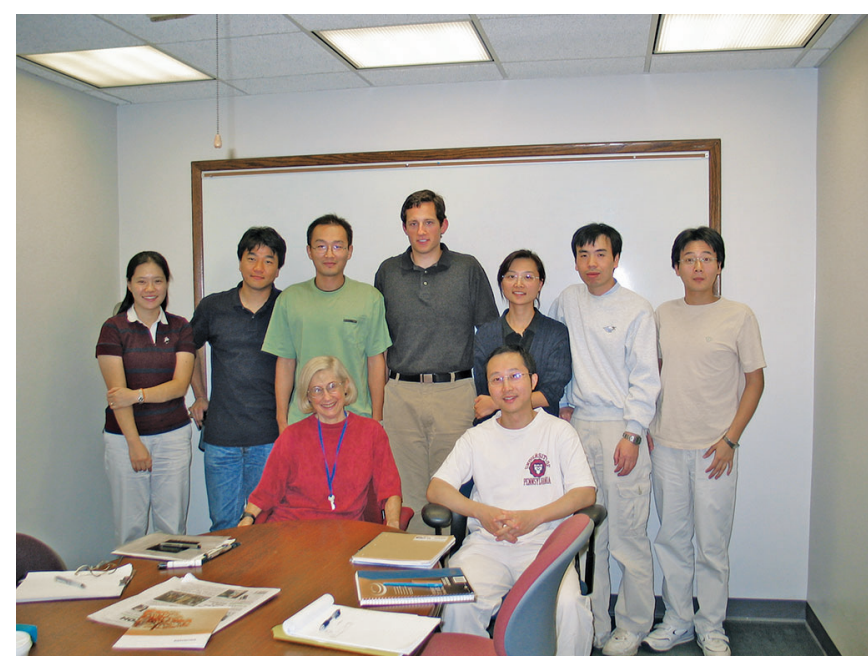

Figure 5. First meeting in the Old Hospital of the Thursday Group, 2004. Back from left: Joung Youn Kim, Yongho Jeon, Fan Lu, John Carew, Hyonho Chun, Xianhong Xie, Weiliang Shi. Front from left: Grace Wahba, Yi Lin.

Gaussian process on $x$ with covariance function $E\left[\eta_{1}(x) \eta_{1}(y)\right]=b R_{J}(x, y)$. Observing $y_{i}=\eta\left(x_{i}\right)+\epsilon_{i}$, $\epsilon_{i} \sim N\left(0, \sigma^{2}\right)$ independent, the posterior mean of $\eta(x)$ is given by $\eta_{\lambda}(x)$, with $\eta_{\lambda}$ minimizing (5) for $\lambda=\sigma^{2} / n b$. See Wahba [12].

For tensor product splines with $\mathcal{N}_{J}=\bigoplus_{\nu}\left\{\phi_{\nu}\right\}$, $J(\eta)=\sum_{\beta} \theta_{\beta}^{-1} J_{\beta}(\eta)$, and $R_{J}=\sum_{\beta} \theta_{\beta} R_{\beta}$, one has $\eta=\sum_{\nu} \psi_{\nu}+\sum_{\beta} \eta_{\beta}$, with $\psi_{\nu}$ diffuse in $\left\{\phi_{\nu}\right\}, \eta_{\beta}$ 's being mean zero Gaussian processes with covariance functions $b \theta_{\beta} R_{\beta}$, independent of each other. The terms $d_{\nu} \phi_{\nu}(x)$ and $\theta_{\beta} \sum_{i=1}^{n} c_{i} R_{\beta}\left(x_{i}, x\right)$ in (9) then give the posterior means of $\psi_{\nu}(x)$ and $\eta_{\beta}(x)$, respectively. See [3, Theorem 3.8].

The correspondence between Bayesian estimation with Gaussian process priors and smoothing with quadratic roughness penalties was first observed by Kimeldorf and Wahba $[4,5]$. An instance of the former is the kriging method widely used in geostatistics, where the covariance function of the Gaussian process is known as the variogram.

Despite the common mathematical structure, the underlying statistical models for spline smoothing and kriging are fundamentally different. With spline smoothing, the true $\eta$ is a smooth function and a comprehensible $J(\eta)$ provides intuitions about what the method does, whereas in kriging the true $\eta$ is a realization of stochastic process and specific models are defined by the variogram.

4.1. Bayesian confidence intervals. Under the Bayes model, $\eta_{\lambda}(x)$ is the posterior mean of $\eta(x)$, and one may also calculate the posterior standard deviation $s(x)$, leading to say a $95 \%$ Bayesian confidence interval $\eta_{\lambda}(x) \pm$ $1.96 s(x)$.

Despite the derivation under the Bayes model, such intervals possess a certain average coverage property in the spline smoothing setting, as shown by Wahba [14] via heuristic arguments and empirical simulations; the coverage percentage over the data points, $\#\left\{\eta\left(x_{i}\right) \in \eta_{\lambda}\left(x_{i}\right) \pm\right.$ $\left.1.96 s\left(x_{i}\right)\right\} / n$, averages to nearly the nominal $95 \%$ over replicates.

For tensor product splines, posterior covariances can be calculated for the $\psi_{\nu}(x)^{\prime}$ s and $\eta_{\beta}(x)^{\prime}$ s, yielding Bayesian confidence intervals for the ANOVA terms. The term-wise intervals do not possess the average coverage property, but do demonstrate favorable behavior such as being tighter where data are dense.

The posterior distributions are with $b=\sigma^{2} / n \lambda$ for fixed $\lambda$. In practice, one selects $\lambda$ by cross validation and also needs an estimate of $\sigma^{2}$.

When $L(\eta)$ in (2) is convex but not quadratic, such as with the logistic regression of Example 1, the respective minimizer $\eta_{\lambda}(x)$ is closer to a posterior mode than a posterior mean; precise Bayesian derivation can be subtle. Substituting $L(\eta)$ by its quadratic approximation at $\eta_{\lambda}$, things reduce to a weighted version of (5), based on which approximate Bayesian confidence intervals can be constructed.

In the Bayesian statistics literature, a Gaussian approximation of the posterior is known as Laplace approximation.

4.2. Restricted maximum likelihood. Under the Bayes model, $\lambda=\sigma^{2} / n b$ is a model parameter via $\sigma^{2}$ and $b$ in the data-generating mechanism, not just an external tuning parameter associated with an estimation method. One may write

$$
\mathbf{y}=S \mathbf{d}+\epsilon^{*}, \quad \epsilon^{*} \sim N\left(\mathbf{0}, \sigma^{2} I+b Q\right),
$$

where $\mathbf{y}, S, \mathbf{d}$, and $Q$ are as in (8).

Let $F$ be $n \times(n-m)$ orthogonal satisfying $F^{T} S=O$. One may obtain the maximum likelihood estimates of $\left(\sigma^{2}, b\right)$ using $F^{T} \mathbf{y} \sim N\left(\mathbf{0}, \sigma^{2} I+b F^{T} Q F\right)$, where the nuisance parameter $\mathbf{d}$ is eliminated. This is known as the restricted maximum likelihood (REML) in the mixed-effect model literature.

In the spline smoothing setting, this approach to the selection of $\lambda$ does not target any statistical loss as cross validation does, but provides a stable empirical performer nevertheless. It makes a good fallback option when effective cross validation scores are not available, say when $\sigma^{2} I$ above is replaced by some non-trivial covariance matrices.

When used in (5), Wahba [15] showed that the REML selection $\lambda_{m}$ cannot exceed the order of $n^{-r /(r+1)}$, achieving a convergence rate no better than

$$
O_{p}\left(n^{-p r /(r+1)}+n^{-r /(r+1)}\right)=O_{p}\left(n^{-r /(r+1)}\right)
$$

by (10); the best possible rate is $O_{p}\left(n^{-p r /(p r+1)}\right)$, however, achievable by the optimally performing GCV score in (15), at $\lambda_{v} \asymp n^{-r /(p r+1)}$. When $p>1$, or the true $\eta$ is "super 
smooth" in Wahba's terms, the REML selection is suboptimal.

4.3. Efficient approximation. For the minimizer $\eta_{\lambda}^{*}$ of (5) in $\mathcal{H}^{*}$ of (11), $\eta_{\lambda}^{*}(x)$ is the posterior mean of $\eta(x)=$ $\eta_{0}(x)+\eta_{1}(x)$, with $\eta_{0}$ diffuse in $\mathcal{N}_{J}$ and $\eta_{1}$ being a mean zero Gaussian process with covariance function $E\left[\eta_{1}(x) \eta_{1}(y)\right]=b R_{J}\left(x, \mathbf{z}^{T}\right) Q^{+} R_{J}(\mathbf{z}, y)$, where $Q$ is $q \times q$ with the $(j, k)$ th entry $R_{J}\left(z_{j}, z_{k}\right)$; see [3, \$3.5.2].

Substituting $R_{J}\left(x, \mathbf{z}^{T}\right) Q^{+} R_{J}(\mathbf{z}, y)$ for $R_{J}(x, y)$ resembles the Nyström matrix approximation, with which a nonnegative definite $K=\left(\begin{array}{ll}A & B^{T} \\ B & C\end{array}\right)$ is to be approximated by $D A^{+} D^{T}$ for $D=\left(\begin{array}{c}A \\ B\end{array}\right)$.

\section{Machine Learning}

The 1990s witnessed the start of some explosive developments in modern machine learning techniques, and the timely publication of Grace's 1990 monograph [16] played a pivotal role in introducing the powerful reproducing kernel Hilbert space machinery to the broader research community. The kernel learning approach bears close resemblance to spline smoothing and kriging. Some classification methods such as the support vector machines can be cast as solutions to regularization problems similar to (2).

Consider pairs of training samples $\left(x_{i}, y_{i}\right)$, where $x_{i} \in$ $x$ are in the feature space and $y_{i}$ are binary class labels. The task is to develop rules to classify future items into one of the two classes based on the features. The performance of classifiers is typically assessed by misclassification rate.

Assuming $y \sim \operatorname{Bin}(1, p(x))$ with $p(x)$ known, the Bayes rule, which minimizes misclassification rate, would classify an item as $y=1$ when $p(x)>0.5$, or $\eta(x)=$ $\log p(x) /(1-p(x))>0$.

It is tempting to employ the logistic regression of Example 1 to estimate the logit $\eta(x)$, then approximate the Bayes rule using $\eta_{\lambda}(x)$ in its place. This is called soft classification by Wahba [17]. Techniques developed for logistic regression aim to minimize a statistical loss

$$
n^{-1} \sum_{i=1}^{n}\left(p_{\lambda}-p\right)\left(x_{i}\right)\left(\eta_{\lambda}-\eta\right)\left(x_{i}\right),
$$

however, which is not directly related to misclassification rate. Also, logistic regression is technically challenged when the true $p(x)$ are mostly near 0 or 1 , that is, when the two labels are well separated in the feature space, which however should be a more favorable environment to train classifiers.

Targeting the Bayes rule directly, one may set $y_{i} \in\{-1,1\}$ and minimize

$$
\frac{1}{n} \sum_{i=1}^{n}\left(1-y_{i} \eta\left(x_{i}\right)\right)_{+}+\lambda J(\eta)
$$

over $\eta \in\{1\} \oplus \mathcal{H}_{J}$, where $(x)_{+}=\max (x, 0)$, and classify a

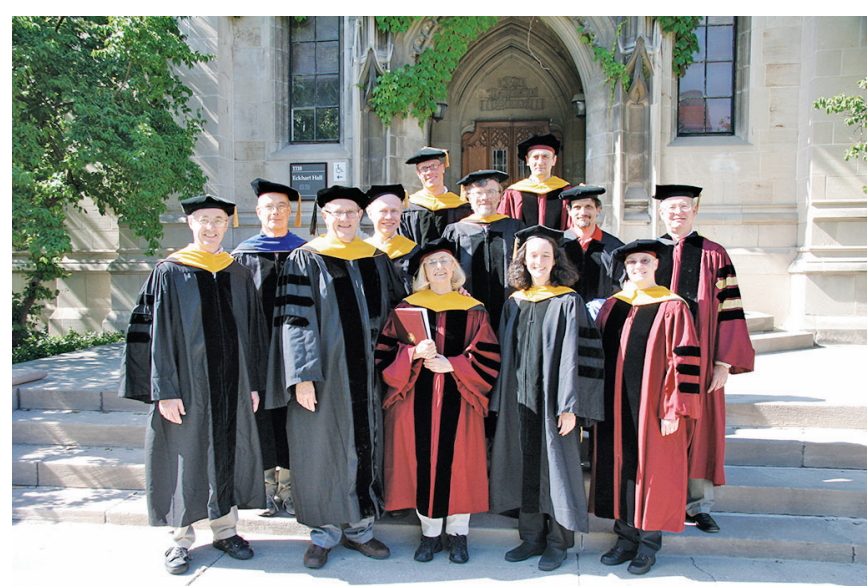

Figure 6. Receiving honorary doctorate from the University of Chicago, 2007. Back from left: Peter McCullagh, Michael Wichura, Stephen Stigler, Per Mykland, Mathias Drton, Greg Lawler, Dan Nicolai, Yali Amit, Ron Thisted. Front from left: Grace Wahba, Mary Sara McPeek, Linda Collins.

future item by $y=\operatorname{sign}\{\eta(x)\}$; this yields a support vector machine. See [17]. Note that the $\eta(x)$ in (17) is not the logit, but defining a classifier in $\operatorname{sign}\{\eta(x)\}$.

When the two types of misclassification incur unequal costs, or when $\sum_{i} I_{\left[y_{i}=1\right]} / \sum_{i} I_{\left[y_{i}=-1\right]}$ is far from the natural ratio due to retrospective sampling, the Bayes rule minimizing the misclassification cost is of the form $\operatorname{sign}\{p(x)-$ $\left.p_{0}\right\}$ for some $p_{0} \neq 0.5$. To target such a rule in the form of $\operatorname{sign}\{\eta(x)\}$, one may modify the terms in (17) as $L\left(y_{i}\right)\left(1-y_{i} \eta\left(x_{i}\right)\right)_{+}$for $L(1)=p_{0}=1-L(-1)$. See [9].

The solution expression in (6) still holds for the minimizer $\eta_{\lambda}$ of (17), though the performance of $\operatorname{sign}\left\{\eta_{\lambda}(x)\right\}$ may not be as sensitive to the selection of $\lambda$. Technical underpinnings, implementation details, and empirical performances can be found in the references cited above, all with Grace among the authors. Extensions of the techniques to multiclass classification are to be found in [7].

\section{Epilogue}

As noted earlier, Grace has worked on a great variety of interesting problems over the years; there was even a Wahba's problem named after her, for her work done while a graduate student at Stanford working part time for IBM [10]. A thorough presentation of the entire body of Grace's life work would be way above our pay grade.

Our attempt in this piece is to provide an overview of a family of spline smoothing techniques that owe their flourishing developments mainly to Grace's ingenuity and persistence. These techniques form a comprehensive, coherent system, anchored in reproducing kernel Hilbert spaces.

Earlier in her career, Grace had also published on approximation theory in the mathematics literature. In recent years, Grace has explored methodologies such as the use of $L_{1}$-type penalties for variable selection and the 
embedding of general discrepancy measures in normed spaces. While a theoretician by training, Grace had a genuine passion for practical applications, and much of her work was motivated by scientific problems in meteorology and the life sciences.

Grace is a member of the National Academy of Sciences since 2000, and received an honorary degree from the University of Chicago in 2007. She is a fellow of the American Academy of Arts and Sciences, the American Association for the Advancement of Science, the American Statistical Association, and the Institute of Mathematical Statistics. Grace was also recognized by the Committee of Presidents of Statistical Societies, receiving the Elizabeth Scott Award in 1996 and the R. A. Fisher Lectureship in 2014. To honor Grace's monumental contributions to statistics and science, a Grace Wahba Award and Lecture was recently established by the Institute of Mathematical Statistics.

\section{References}

[1] N. Aronszajn, Theory of reproducing kernels, Trans. Amer. Math. Soc. 68 (1950), 337-404, DOI 10.2307/1990404. MR51437

[2] P. Craven and G. Wahba, Smoothing noisy data with spline functions. Estimating the correct degree of smoothing by the method of generalized cross-validation, Numer. Math. 31 $(1978 / 79)$, no. 4, 377-403, DOI 10.1007/BF01404567. MR516581

[3] C. Gu, Smoothing spline ANOVA models, 2nd ed., Springer Series in Statistics, vol. 297, Springer, New York, 2013, DOI 10.1007/978-1-4614-5369-7. MR3025869

[4] G. Kimeldorf and G. Wahba, A correspondence between Bayesian estimation on stochastic processes and smoothing by splines, Ann. Math. Statist. 41 (1970), 495-502, DOI 10.1214/aoms/1177697089. MR254999

[5] G. Kimeldorf and G. Wahba, Spline functions and stochastic processes, Sankhyā Ser. A 32 (1970), 173-180. MR303594

[6] G. Kimeldorf and G. Wahba, Some results on Tchebycheffian spline functions, J. Math. Anal. Appl. 33 (1971), 82-95, DOI 10.1016/0022-247X(71)90184-3. MR290013

[7 Y. Lee, Y. Lin, and G. Wahba, Multicategory support vector machines: theory and application to the classification of microarray data and satellite radiance data, $\mathrm{J}$. Amer. Statist. Assoc. 99 (2004), no. 465, 67-81, DOI 10.1198/016214504000000098. MR2054287

[8] K.-C. Li, Asymptotic optimality of $C_{L}$ and generalized crossvalidation in ridge regression with application to spline smoothing, Ann. Statist. 14 (1986), no. 3, 1101-1112, DOI 10.1214/aos/1176350052, MR856808

[9] Y. Lin, G. Wahba, H. Zhang, and Y. Lee, Properties and adaptive tuning of support vector machines, Mach. Learn. 48 (2002), 115-136.

[10] D. Nychka, P. Ma, and D. Bates, A conversation with Grace Wahba, Statist. Sci. 35 (2020), no. 2, 308-320, DOI 10.1214/19-STS734. MR4106607
[11] B. W. Silverman, On the estimation of a probability density function by the maximum penalized likelihood method, Ann. Statist. 10 (1982), no. 3, 795-810. MR663433

[12] G. Wahba, Improper priors, spline smoothing and the problem of guarding against model errors in regression, J. Roy. Statist. Soc. Ser. B 40 (1978), no. 3, 364-372. MR522220

[13] G. Wahba, Spline interpolation and smoothing on the sphere, SIAM J. Sci. Statist. Comput. 2 (1981), no. 1, 5-16, DOI 10.1137/0902002 MR618629

[14] G. Wahba, Bayesian "confidence intervals" for the crossvalidated smoothing spline, J. Roy. Statist. Soc. Ser. B 45 (1983), no. 1, 133-150. MR701084

[15] G. Wahba, A comparison of GCV and GML for choosing the smoothing parameter in the generalized spline smoothing problem, Ann. Statist. 13 (1985), no. 4, 1378-1402, DOI 10.1214/aos/1176349743. MR811498

[16] G. Wahba, Spline models for observational data, CBMSNSF Regional Conference Series in Applied Mathematics, vol. 59, Society for Industrial and Applied Mathematics (SIAM), Philadelphia, PA, 1990, DOI 10.1137/1.9781611970128, MR1045442

[17] G. Wahba, Soft and hard classification by reproducing kernel Hilbert space methods, Proc. Natl. Acad. Sci. USA 99 (2002), no. 26, 16524-16530, DOI 10.1073/pnas.242574899. MR1947755

[18] G. Wahba, Y. Wang, C. Gu, R. Klein, and B. Klein, Smoothing spline ANOVA for exponential families, with application to the Wisconsin Epidemiological Study of Diabetic Retinopathy, Ann. Statist. 23 (1995), no. 6, 1865-1895, DOI $10.1214 / \mathrm{aos} / 1034713638$, MR1389856

[19] G. Wahba and J. Wendelberger, Some new mathematical methods for variational objective analysis using splines and cross validation, Monthly Weather Rev. 108 (1980), 1122-1145.

[20] Y. Wang, Smoothing splines: Methods and applications, Monographs on Statistics and Applied Probability, vol. 121, CRC Press, Boca Raton, FL, 2011, DOI 10.1201/b10954, MR2814838

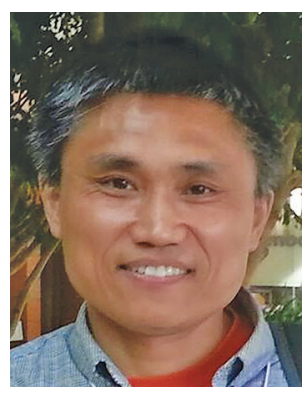

Chong Gu

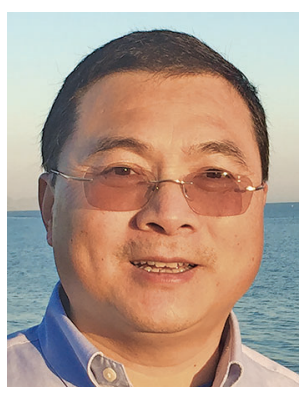

Yuedong Wang

\section{Credits}

Opening photo is courtesy of Chong Gu.

Figures $1-3$ are courtesy of the authors.

Figures 4 and 5 are courtesy of Grace Wahba.

Figure 6 is courtesy of Stephen M. Stigler.

Author photos are courtesy of the authors. 